\title{
Effect of enhanced expression of COL8A1 on lymphatic metastasis of hepatocellular carcinoma in mice
}

\author{
ZHEN-HAI MA $^{1 *}$, JIN-HUI MA $^{1^{*}}$, LI JIA $^{2}$ and YONG-FU ZHAO ${ }^{1}$ \\ ${ }^{1}$ Department of General Surgery, The Second Affiliated Hospital of Dalian Medical University, Dalian 116027; \\ ${ }^{2}$ College of Laboratory Medicine, Dalian Medical University, Dalian 116044, P.R. China
}

Received April 26, 2012; Accepted July 25, 2012

DOI: $10.3892 /$ etm.2012.652

\begin{abstract}
The present study aimed to investigate the influence of COL8A1 expression on cell invasiveness, drug sensitivity and tumorigenicity of hepatocellular carcinoma Hepa1-6 cells with low metastatic potential. COL8A1-1-pEGFP-N2 and pEGFP-N2 were transfected into experimental and control group cells. The COL8A1 expression in transfected Hepa1-6 cells was analyzed with RT-PCR and western blot analysis. The invasive potential of transfected Hepa1- 6 cells was tested in invasion experiments in vitro and the tumorigenic ability of the transfected Hepa1-6 cells was tested in mouse tumors in vivo. Hepa1-6 cell proliferation and D-limonene sensitivity was analyzed using the MTT method. Expression of COL8A1 in the Hepa1-6/COL8A1 group showed a significant increase when compared with the untransfected cells of the Hepa1-6 control group and empty-plasmid transfected cells from the Hepa1-6/mock control group. Enhanced COL8A1 expression increased cell proliferation and matrix adhesion ability via invasion and tumorigenesis in vivo while the sensitivity to D-limonene was concurrently inhibited. The expression of COL8A1 in hepatocarcinoma cells was correlated with increased tumor cell proliferation, invasion, in vivo tumorigenicity and reduced antitumor drug sensitivity, and may provide novel targets for tumor therapy.
\end{abstract}

Correspondence to: Professor Yong-Fu Zhao, Department of General Surgery, The Second Affiliated Hospital of Dalian Medical University, Dalian 116027, P.R. China

E-mail: zyf0386@sina.com

${ }^{*}$ Contributed equally

Abbreviations: COL8A1, type VIII collagen one; FBS, phosphate buffered saline; SDS-PAGE, sodium dodecyl sulfate polyacrylamide gel electrophoresis; RT-PCR, reverse transcription-polymerase chain reaction; MTT, 3-(4,5)-dimethylthiazol (-2-yl)-2,5 diphenyltetrazolium bromide

Key words: hepatocarcinoma, COL8A1, D-limonene, lymphatic metastasis

\section{Introduction}

Hepatocarcinoma (liver cancer) is the third-leading cause of cancer-related mortality and the fifth most common malignancy worldwide (1). In China presently, hepatocarcinoma is the second major cause of cancer-related mortalities, with a mortality rate of 26.26 per 100,000 individuals (males, 37.55; and females, 14.45 per 100,000), accounting for $19.33 \%$ of all types of cancers. Accordingly, the estimated annual incidence of cases and mortalities from hepatocarcinoma are 360,000 and 350,000 , respectively $(2,3)$. Type VIII collagen, a non-fibrillar short-chain collagen, is a structural component of numerous extracellular matrices $(4,5)$. It is highly expressed by vascular smooth muscle cells and is considered to be a key component in vascular remodeling $(6,7)$. Type VIII collagen exists as a heterodimer composed of two distinct $\alpha$-chains (COL8A1 and COL8A2), each with a molecular weight of approximately $60 \mathrm{kDa}(8)$. The high expression level of COL8A1 in vascular endothelial cells and tumor cells has gained extensive attention $(9,10)$. High expression of COL8A1 in tumor cells may be associated with tumor cell proliferation. The present study focused on the role of COL8A1 in the tumor metastasis of liver cancer, which may provide experimental basis for research and development of antitumor drugs.

\section{Materials and methods}

Cell culture. The mouse hepatocarcinoma cell line Hepa1-6, which lacks metastatic potential in the lymph nodes (provided by the Cell Center of Peking Union Medical College, China) was cultured in DMEM medium (HyClone, Logan, UT, USA), supplemented with 10\% FBS (HyClone), streptomycin $100 \mathrm{U} / \mathrm{ml}$, penicillin $100 \mathrm{U} / \mathrm{ml}$. The cells were incubated in a humidified atmosphere containing $5 \% \mathrm{CO}_{2}$ at $37^{\circ} \mathrm{C}$ for $24 \mathrm{~h}$. The cells were in logarithmic growth phase.

Cell transfection. COL8A1-1-pEGFP-N2 and pEGFP-N2 were transfected, into the experimental and control group cells, respectively. The specific steps were as follows. i) Hepa1-6 cells were cultured in DMEM culture medium for $24 \mathrm{~h}$, up to $40-80 \%$ confluency in cell fusion. ii) Carrier $(6 \mu \mathrm{g})$ was dissolved in DMEM culture medium (without FBS and antibiotics) and when the volume reached $300 \mu \mathrm{l}, 50 \mu \mathrm{l}$ liposome reagent was added, mixed well and then kept at room 
temperature for $5 \mathrm{~min}$, and ultimately reaction complexes were formed. iii) Hepa1-6 cells were prepared by adding $7 \mathrm{ml}$ DMEM medium. Inclusion of such a complex containing the reaction of the test tube and $1 \mathrm{ml}$ DMEM was mixed well and immediately transferred onto a dish. Cells were incubated in a humidified atmosphere containing $5 \% \mathrm{CO}_{2}$ at $37^{\circ} \mathrm{C}$ for $8 \mathrm{~h}$ by replacing the fluid for normal cell growth and continuing for an additional $48 \mathrm{~h}$. The experimental group cells were cultured in $\mathrm{G} 418(800 \mu \mathrm{g} / \mathrm{ml})$ selective medium, and the control group of untransfected Hepa1- 6 cells were cultured in G418 medium. Cells were cultured in the G418 $(400 \mu \mathrm{g} / \mathrm{ml})$ selective medium until the majority of the untransfected cells were dead in the control group and the transfected cells in the experimental group were cloned. The selective medium was replaced every 3 days for 20 days. A single clonal cell was picked for screening into 24-well plates using the cloning ring method together with cultured cells in G418 $(400 \mu \mathrm{g} / \mathrm{ml})$ selective medium.

RT-PCR analysis. For RT-PCR analysis of COL8A1 mRNA levels, total RNA was isolated from cells using TRIzol (Invitrogen, Carlsbad, CA, USA), and cDNA was synthesized using an RT-PCR kit (Takara, Shiga, Japan) according to the manufacturer's instructions. The primer sequences were as follows; 5'-GCTGCTGGGAATACTGTTCA3'(F) and 5'-GGGAGGTATGGGTACTCTTT-3'(R) for COL8A1; 5'-GGCCGTGAAGTCGTCAGAAC-3'(F) and 5'-GCCACGATGCCCAGGAA-3'(R) for GAPDH. PCR analysis was performed under the following conditions: denaturation at $94^{\circ} \mathrm{C}$ for $1 \mathrm{~min}$, and 30 cycles of denaturation for $20 \mathrm{sec}$ at $97^{\circ} \mathrm{C}$, annealing for $20 \mathrm{sec}$ at $64^{\circ} \mathrm{C}$ and extension for $20 \mathrm{sec}$ at $72^{\circ} \mathrm{C}$. The amplified products were analyzed by agarose gel electrophoresis using $1.0 \%$ gel, followed by ethidium bromide staining.

Western blot analysis. Western blot analysis was performed to evaluate COL8A1 protein levels. The protein was harvested from cells using a $2 \mathrm{X}$ concentrated electrophoresis sample buffer (125 mmol/1 Tris-HCl, pH 6.8, 2\% sodium dodecyl sulfate, $5 \%$ glycerol and $1 \% \beta$-mercaptoethanol). Equal amounts of denatured proteins $(35 \mu \mathrm{g})$ were resolved by $10 \%$ SDS-PAGE and then blotted onto PVDF membranes (Pall Corporation). After blocking for $2 \mathrm{~h}$ with phosphate-buffered saline containing $0.1 \%$ Tween 20 and $5 \%$ powdered skimmed milk, the blots were incubated with rabbit anti-mouse COL8A1 polyclonal antibodies (Abcam, Cambridge, UK; ab58776, 1:200 dilution) overnight in $5 \%$ powdered skimmed milk buffer, washed thrice with phosphate-buffered saline with $0.1 \%$ Tween 20 and then incubated with secondary antibody anti-rabbit-HRP (Santa Cruz Biotechnology Inc., Santa Cruz, CA, USA; 1:3000 dilution). GAPDH antibody (Santa Cruz Biotechnology, Inc.; 1:200 dilution) was used as a control. All bands were detected using ECL western blot kit (Amersham Biosciences, Buckinghamshire, UK).

MTT assay. Hepa1-6, Hepa1-6/COL8A1 and Hepa1-6/mock cells $\left(1 \times 10^{6}\right)$ in $200 \mu \mathrm{l}$ RPMI-1640 were seeded in duplicate into each well of 96-well culture plates, and $100 \mu \mathrm{l}$ MTT (5 mg/ml, Sigma, St. Louis, MO, USA) was added at 24, 48, 72 , 96 and $120 \mathrm{~h}$. After a 4-h incubation at $37^{\circ} \mathrm{C}$ in $5 \% \mathrm{CO}_{2}, 100 \mu \mathrm{l}$ DMSO (Gibco, Carlsbad, CA, USA) was pipetted to solubi- lize the formazan product for $30 \mathrm{~min}$ at room temperature. The absorbancy of A570 was measured using a microplate reader (Bio-Rad, Hercules, CA, USA). Growth rate $(\%)=$ A570(Hepa1-6/COL8A1) $\div$ A570(Hepa1-6) x 100\%.

In vitro ECM invasion assay. Cell invasion in vitro was demonstrated using 24-well Transwell units (Corning, NY, USA) with $8-\mu \mathrm{m}$ pore size polycarbonate filter coated with ECMatrix gel (Chemicon, Temecula, CA, USA) to form a continuous thin layer. Hepa1-6, Hepa1-6/COL8A1 and Hepa1-6/mock cells $\left(3 \times 10^{5}\right)$ were harvested in RPMI-1640 containing $1 \%$ FBS and added to the upper chamber. The lower chamber was filled with $500 \mu 1$ RPMI-1640 containing $10 \%$ FBS. The cells were incubated for $24 \mathrm{~h}$ at $37^{\circ} \mathrm{C}$ with $5 \% \mathrm{CO}_{2}$. At the end of incubation, the cells on the upper surface of the filter were completely removed by wiping with a cotton swab. The filters were then fixed in methanol and were stained with Wright-Giemsa. The number of cells that had invaded the Matrigel and reached the lower surface of the filter were counted under a light microscope at a magnification of $\mathrm{x} 200$. Triplicate samples were acquired, and the data were expressed as the average cell number of 5 fields. Invasive cells were calculated and analyzed with the Image-Pro Plus 4.5 software (Media Cybernetics).

In vivo tumorigenicity analysis. A total of $60 \mathrm{C} 57 \mathrm{~L}$ mice (provided by Dalian Medical University, Dalian, China) were randomly divided into three groups, each group having 20 mice. The logarithmic phase Hepa1-6, Hepa1-6/COL8A1 and Hepa1-6/mock cells were injected subcutaneously in mice. The mice were sacrificed three weeks later. Swollen axillary lymph nodes, fixed with $4 \%$ formaldehyde from the 3 groups were generally compared for tumor weight and metastatic rates. Paraffin sections, hematoxylin and eosin staining and observation of tumor cells were performed under a microscope.

Drug sensitivity assay. To assess chemosensitivity to D-limonene (Sigma); Hepa1-6, Hepa1-6/COL8A1 and Hepa1-6/mock cells $\left(3 \times 10^{5}\right)$ cultured for $24 \mathrm{~h}$, were incubated with various concentrations of D-limonene $(0,0.2,0.4,0.8$ and $1.6 \mathrm{~g} / \mathrm{ml})$ for another $48 \mathrm{~h}$. Then cells were treated with MTT as described previously and each group contained three wells. Cell survival rate $(\%)=$ A570(D-limonene $\left.{ }^{+}\right) \div$A570(D-limonene $)$x 100 .

Statistical analysis. SPSS 14.0 software (SPSS, Chicago, IL, USA) was used. Each assay was performed at least three times. The data are expressed as mean \pm SD and the Student's $\mathrm{t}$-test was used to determine the significance of differences in multiple comparisons. $\mathrm{P}<0.05$ was considered to indicate a statistically significant result.

\section{Results}

cDNA transfection increases the expression of COL8A1. In order to verify whether stable expression of COL8A1 affects hepatocarcinoma tumors in mice, we transfected Hepa1-6 cells that expressed COL8A1 at a low level with the constructed plasmid COL8A1-pEGFP-N2. After 3 weeks, we analyzed the stable transfected Hepa1-6/COL8A1 cells by RT-PCR and western blot analysis. We set two negative control groups; 

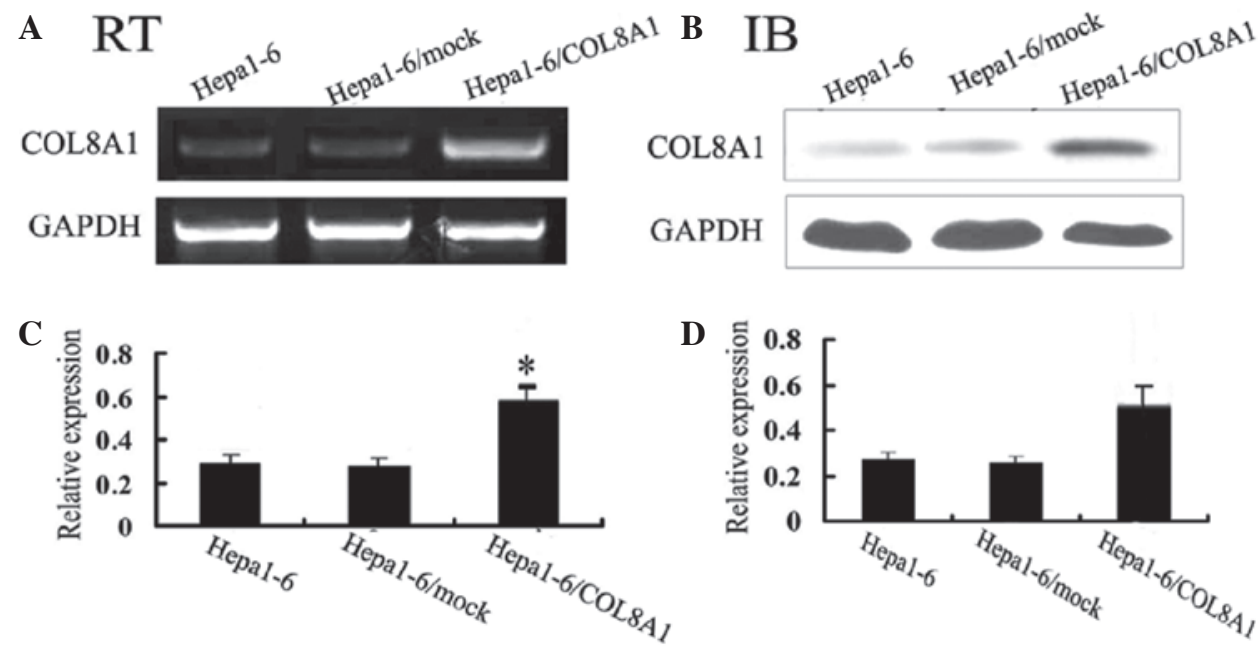

Figure 1. Stable expression of COL8A1 in Hepa1-6 cells. Hepa1-6 cells were transfected with a GFP-tagged COL8A1 expression vector. (A) Total RNA was extracted and analyzed by RT-PCR using primers specific to COL8A1 and GAPDH. (B-D) Hepa1-6, GFP-vector control and Hepa1-6/COL8A1 cells were lysed, and the expression of COL8A1 was analyzed by western blot analysis using anti-COL8A1 antibody. Relative signal intensities of COL8A1 mRNA, and protein levels were normalized against those of GAPDH. RT-PCR, reverse transcription-polymerase chain reaction.

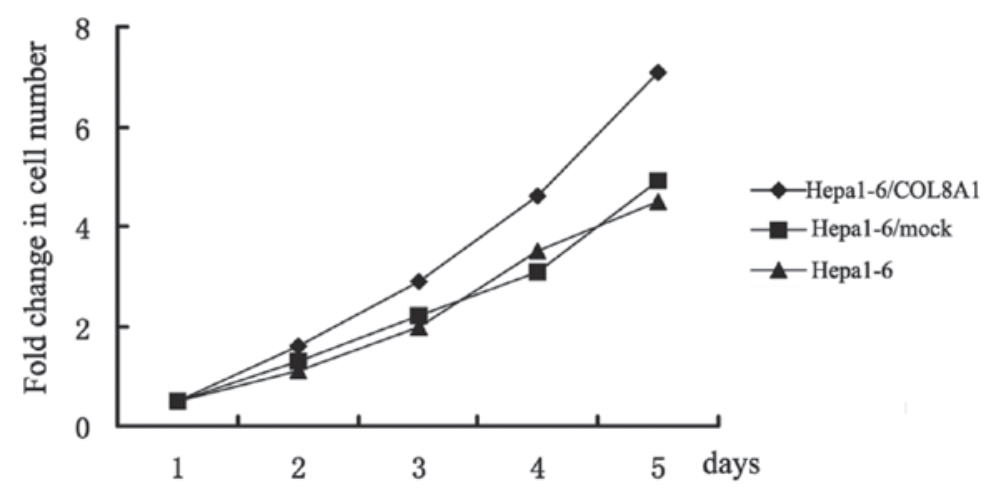

Figure 2. Stable expression of COL8A1 increases Hepa1-6/COL8A1 cell proliferation in vitro. Cells were harvested at 1,2,3,4 and 5 days post-transfection and cell proliferation was measured by MTT assay. Increased growth was detected in Hepa1-6/COL8A1 cells compared with control cells. The data was obtained from three independent experiments.

i) nontransfected Hepa1-6 cells; ii) empty plasmid-transfected Hepa1-6/mock cells, through which transfection of Hepa1-6 cells was observed. Following stable transfection, Hepa1-6/ COL8A1 cells highly expressed COL8A1 and the other two control groups expressed COL8A1 at lower levels (Fig. 1). These results confirmed that it was feasible, reliable and effective to apply this method of stable transfection.

Enhanced COL8A1 expression increases Hepal-6 cell proliferation in vitro. After enhancing the expression of the COL8A1 gene in Hepa1-6 cells, we determined the cell proliferation using MTT at 24, 48, 72, 96 and $120 \mathrm{~h}$, and also set a control group of untransfected Hepa1- 6 cells and another of empty plasmid-transfected Hepa1-6/mock cells. The results demonstrated that the growth rate curve of Hepa1-6/COL8A1 cells increased, while the growth rate curves of the control group exhibited a flat trend. Therefore, enhancing COL8A1 expression increased the proliferative ability of Hepa1-6 cells in vitro (Fig. 2).
Enhancing COL8A1 expression increases Hepal-6/COL8A1 cell invasion in vitro. In order to verify whether the stable expression of COL8A1 plays a key role in Hepa1-6/COL8A1 cell invasion in vitro we compared the cell proliferation of Hepa1-6/COL8A1 cells of the experimental group with that of the two control groups: the untransfected cells of the Hepa1-6 control group and the empty plasmid-transfected cells of the Hepa1-6/mock control group (Fig. 3A). The number of cells that crossed the membrane was higher in the experimental group $(21 \pm 3)(\mathrm{P}<0.05$; Fig. $3 \mathrm{~B})$. These results indicate that stable expression of COL8A1 is able to promote the in vitro cell invasion of Hepa1-6/COL8A1 cells and plays a key role in cancer metastasis.

Enhanced COL8A1 expression promotes the tumorigenicity of Hepal-6 cells. The tumor formation experiment in vivo (Fig. 4A) showed that, compared with the control groups of untransfected Hepa1-6 cells and empty plasmid-transfected Hepa1-6 cells, the experimental group Hepa1-6/COL8A1 cells 
$\mathbf{A}$

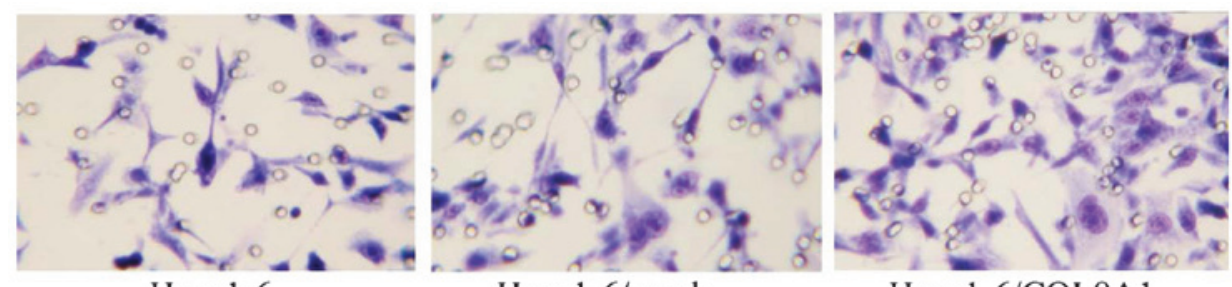

Hepal-6

Hepal-6/mock

Hepal-6/COL8A1

B

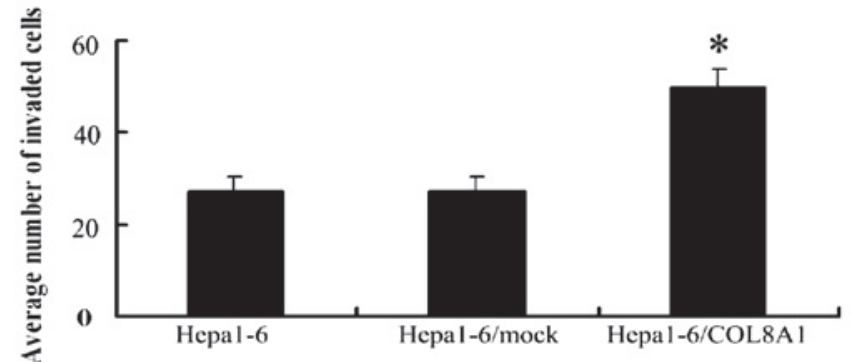

Figure 3. Stable expression of COL8A1 affects the invasive capability of Hepa1-6/COL8A1 cells. ECMatrix gel analysis in vitro was performed. (A) The Wright-Giemsa staining results of the lower surface filters show that the cells had invaded through the filter and attached to the lower side (x400). The number of invasive Hepa1-6/COL8A1 cells was higher than the numbers of Hepa1-6 and Hepa1-6/mock cells. (B) The average number of cells that invaded through the filter was calculated. Hepa1-6/COL8A1 cells were significantly more invasive ( $\mathrm{P}<0.05)$ than controls. The data were obtained from three independent experiments.

A

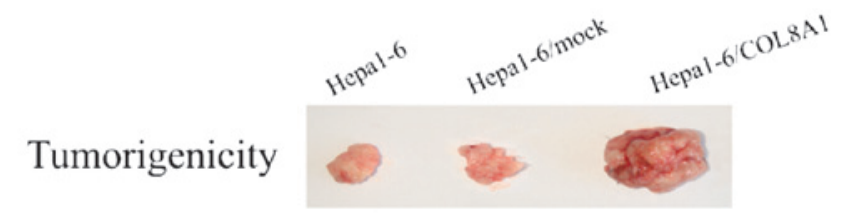

B

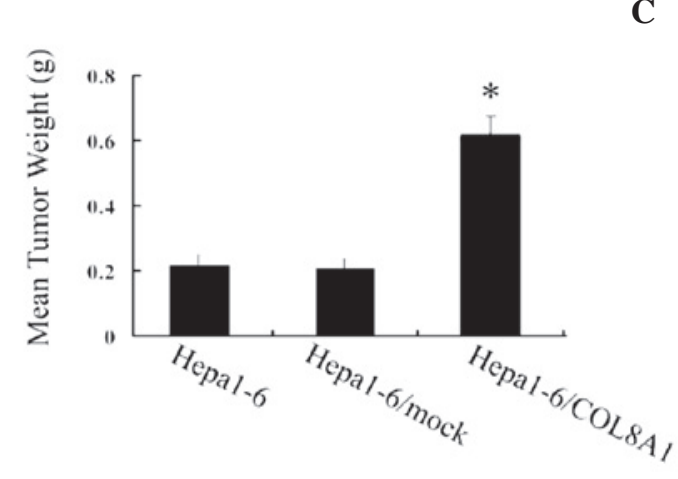

C

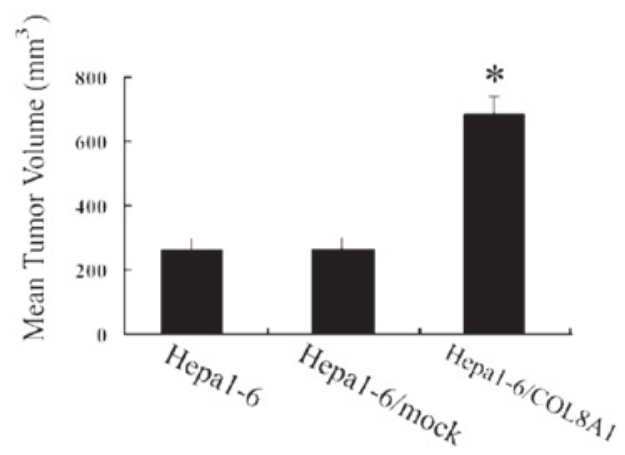

Figure 4. Stable expression of COL8A1 in Hepa1-6 cells increases tumorigenicity in nude mice. (A) Three groups of nude mice were injected subcutaneously with Hepa1-6, Hepa1-6/mock and Hepa1-6/COL8A1 cells. After 3 weeks, tumors were excised, weighed, sized and photographed. (B and C) Hepa1-6/COL8A1 groups showed a significant increase in mean tumor weight $(\mathrm{n}=10)$ and volume $\left(\mathrm{n}=10 ;{ }^{*} \mathrm{P}<0.05\right)$, compared with control groups, respectively. The data were obtained from three independent experiments.

clearly exhibited tumor formation. These results indicate that the stable expression of COL $8 \mathrm{~A} 1$ promotes the tumorigenicity of Hepa1-6/COL8A1 cells.

Increased COL8A1 expression reduces the sensitivity of Hepal-6 cells to D-limonene treatment. Compared with the control group of untransfected Hepa1-6 cells and empty plasmid-transfected Hepa1-6/mock cells, increased COL8A1 expression inhibited the sensitivity of Hepa1-6/COL8A1 cells to D-limonene treatment.

\section{Discussion}

One of the main purposes of cancer research is to identify the genetic factors that contribute to tumor formation and to elucidate the mechanism of tumor development. Therefore, certain specific gene proteins may be investigated due to their potential role as markers of tumor progression or as a treatment goal. It has been reported that at initial diagnosis, the cancer in over $60 \%$ of patients has progressed. Metastasis is a characteristic of malignant tumors and is the main cause of mortality in 


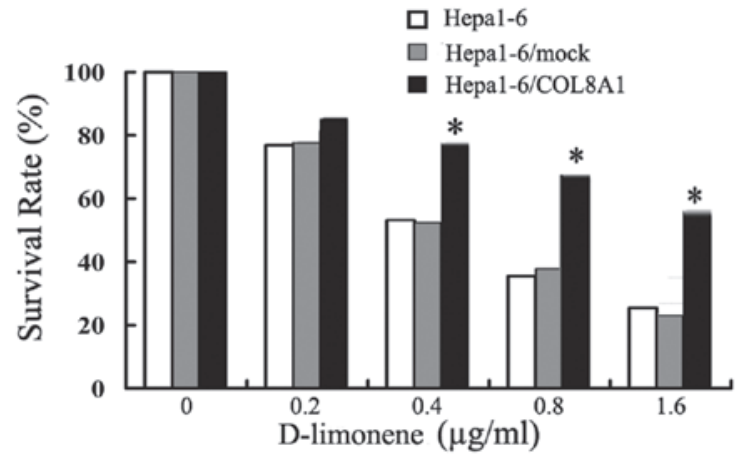

Figure 5. Stable expression of COL8A1 inhibits the sensitivity of Hepa1-6 cells to D-limonene treatment. Hepa1-6, Hepa1-6/mock and Hepa1-6/COL8A1 cells were treated with various concentrations of D-limonene. Cell viability was determined by MTT chronometry. Cell survival rate (\%) was calculated as A570 (D-limonene $\left.{ }^{+}\right) \div$A570 (D-limonene $\left.{ }^{-}\right)$x $100 \%$. The data were obtained from three independent experiments $\left({ }^{*} \mathrm{P}<0.05\right)$.

patients with malignant tumors. Elucidation of the mechanisms involved in tumor metastasis has great theoretical significance and practical value. The formation and development of tumors involves cell proliferation, differentiation disorders, apoptosis, an abnormal angiogenesis pathway and numerous other factors. It is a complex process involving multiple genetic changes.

Currently, increasing attention has been paid to COL8A1 gene due to its expression in a number of rapidly proliferating cell types $(10,11)$. COL8A1 which is located around the cell may play a sustaining role in the cell proliferative stage. COL8A1 has also been known to take on the role of chemokine stromal cells, including smooth muscle cells, and provides a substrate to enhance cell transfer (12-14). COL8A1 gene expression profiles divide gastrointestinal stromal tumors into different tumor groups (9). D-limonene is an available monomer composition which is extracted from traditional Chinese medicinal orange peel and belongs to the natural monoterpene set of compounds. Due to its citrus aroma, D-limonene is widely used as an additive in perfumes, soap, food, chewing gum and drinks (15). A large number of experiments have proven that D-limonene exhibits anticancer activity and plays a preventive and therapeutical role in rodent gastric, breast, liver, colon, pancreatic and skin cancer, and other tumors which are chemically induced. Its mechanisms include direct cytotoxicity to tumor cells, inducing tumor cell death and inhibition of the enzyme activity and levels of matrix protein in tumor cells (16-20). D-limonene also inhibits the formation of tumor microvasculature and lymphatic tubes, resulting in low microvascular density. Thus tumor metastasis was found to be inhibited (21-23).

In the present study, we transfected a constructed plasmid of COL8A1-pEGFP-N2 to low COL8A1-expressing Hepa1-6 cells and analyzed the stably transfected Hepa1-6/COL8A1 cells using RT-PCR and western blot analysis. Following stable transfection, Hepa1-6/COL8A1 cells expressed COL8A1 at high levels, and the other two control groups expressed COL8A1 at low levels. These results sufficiently demonstrated that it was feasible, reliable and effective for us to perform this method of stable transfection.

After using the cDNA transfection technique to upregulate COL8A1 expression, the cell proliferation and invasion of Hepa1-6/COL8A1 cells increased. The tumorigenicity in vivo trials demonstrated that the tumorigenicity of Hepa1-6/ COL8A1 cells was clear $(\mathrm{P}<0.05)$. The stable expression of COL8A1 promoted the tumorigenicity of Hepa1-6/COL8A1 cells, which indicated that enhanced expression of COL8A1 affected Hepa1-6/COL8A1 cell in vivo tumorigenicity.

In conclusion, our results suggest that COL8A1 expression is closely related to tumor cell proliferation, invasion and tumorigenicity in vivo. COL8A1 overexpression inhibits drug sensitivity as determined by the effects of D-limonene on Hepa1-6/COL8A1 cells. COL8A1 expression may be one of the key mechanisms in the regulation of cytokines in tumor metastasis. Increased COL8A1 expression enhances tumorigenicity of the cells in the body, which provides new targets for the diagnosis and treatment of over $60 \%$ of the cancer patients lymph node metastasis. It also provides valuable experimental evidence and clues for the design of anticancer drugs.

\section{Acknowledgements}

This work was supported by the Science Foundation of Liaoning Province (no. 20092164), the Creating Team Item of Liaoning Province (no. 2008T033), China.

\section{References}

1. Andrisani OM, Studach L and Merle P: Gene signatures in hepatocellular carcinoma (HCC). Semin Cancer Biol 21: 4-9, 2011.

2. Chen JG, Zhang SW and Chen WQ: Analysis of liver cancer mortality in the national retrospective sampling survey of death causes in China, 2004-2005. Zhonghua Yu Fang Yi Xue Za Zhi 44: 383-389, 2010 (In Chinese).

3. Wang Y, Zhang Y, Peitgen HO, Schenk A, Yuan L, Wei G and Sun Y: Precise local resection for hepatocellular carcinoma based on tumor-surrounding vascular anatomy revealed by $3 \mathrm{D}$ analysis. Dig Surg 29: 99-106, 2012.

4. Muragaki Y, Shiota C, Inoue M, Ooshima A, Olsen BR and Ninomiya Y: alpha 1(VIII)-collagen gene transcripts encode a short-chain collagen polypeptide and are expressed by various epithelial, endothelial and mesenchymal cells in newborn mouse tissues. Eur J Biochem 207: 895-902, 1992.

5. Kittelberger R, Davis PF, Flynn DW and Greenhill NS: Distribution of type VIII collagen in tissues: an immunohistochemical study. Connect Tissue Res 24: 303-318, 1990.

6. Hou G, Mulholland D, Gronska MA and Bendeck MP: Type VIII collagen stimulates smooth muscle cell migration and matrix metalloproteinase synthesis after arterial injury. Am J Pathol 156: 467-476, 2000.

7. Adiguzel E, Hou G, Mulholland D, Hopfer U, Fukai N, Olsen B and Bendeck M: Migration and growth are attenuated in vascular smooth muscle cells with type VIII collagen-null alleles. Arterioscler Thromb Vasc Biol 26: 56-61, 2006.

8. Zhao Y, Jia L, Mao X, Xu H, Wang B and Liu Y: siRNA-targeted COL8A1 inhibits proliferation, reduces invasion and enhances sensitivity to D-limonene treatment in hepatocarcinoma cells. IUBMB Life 61: 74-79, 2009.

9. Kapoor R, Sakai LY, Funk S, Roux E, Bornstein P and Sage EH: Type VIII collagen has a restricted distribution in specialized extracellular matrices. J Cell Biol 107: 721-730, 1988.

10. Koon N, Schneider-Stock R, Sarlomo-Rikala M, Lasota J, Smolkin M, Petroni G, Zaika A, Boltze C, Meyer F, Andersson L, Knuutila S, Miettinen M and El-Rifai W: Molecular targets for tumour progression in gastrointestinal stromal tumours. Gut 53: 235-240, 2004.

11. Illidge C, Kielty CM and Shuttleworth CA: Stability of type VIII collagen homotrimers: comparison with alpha1(X). Biochem Soc Trans 26: S18, 1998

12. Yasuda O, Zhang SH, Miyamoto Y and Maeda N: Differential expression of the alpha1 type VIII collagen gene by smooth muscle cells from atherosclerotic plaques of apolipoprotein-E-deficient mice. J Vasc Res 37: 158-169, 2000. 
13. Plenz GM, Deng MC, Robenk H and Völker W: Vascular collagens: spotlight on the role of type VIII collagen in atherogenesis. Atherosclerosis 166: 1-11, 2003.

14. Alitalo K, Bornstein P, Vaheri A and Sage H: Biosynthesis of an unusual collagen type by human astrocytoma cells in vitro. J Biol Chem 258: 2653-2661, 1983.

15. Crowell PL: Prevention and therapy of cancer by dietary monoterpenes. J Nutr 129: 775S-778S, 1999.

16. Satomi Y, Ohara K, Yazaki K, Ito M, Honda G and Nishino $\mathrm{H}$ : Production of the monoterpene limonene and modulation of apoptosis-related proteins in embryonic-mouse NIH 3T3 fibroblast cells by introduction of the limonene synthase gene isolated from Japanese catnip (Schizonepeta tenuifolia). Biotechnol Appl Bioch 52: 185-190, 2009.

17. Tsuda H, Ohshima Y, Nomoto H, Fujita K, Matsuda E, Iigo M Takasuka $\mathrm{N}$ and Moore MA: Cancer prevention by natural compounds. Drug Metab Pharmacokinet 19: 245-263, 2004.

18. Mo H and Elson CE: Studies of the isoprenoid-mediated inhibition of mevalonate synthesis applied to cancer chemotherapy and chemoprevention. Exp Biol Med (Maywood) 229: 567-585, 2004.
19. Van der Logt EM, Roelofs HM, van Lieshout EM, Nagengast FM and Peters WH: Effects of dietary anticarcinogens and nonsteroidal anti-inflammatory drugs on rat gastrointestinal UDP-glucuronosyltransferases. Anticancer Res 24: 843-849, 2004.

20. Del Toro-Arreola S, Flores-Torales E, Torres-Lozano C, Del Toro-Arreola A, Tostado-Pelayo K, Guadalupe RamirezDueñas $M$ and Daneri-Navarro A: Effect of D-limonene on immune response in $\mathrm{BALB} / \mathrm{c}$ mice with lymphoma. Int Immunopharmacol 5: 829-838, 2005.

21. Ji J, Zhang L, Wu YY, Zhu XY, Lv SQ and Sun XZ: Induction of apoptosis by d-limonene is mediated by a caspase-dependent mitochondrial death pathway in human leukemia cells. Leuk Lymphoma 47: 2617-2624, 2006.

22. Lu XG, Zhan LB, Feng BA, Qu MY, Yu LH and Xie JH: Inhibition of growth and metastasis of human gastric cancer implanted in nude mice by d-limonene. World J Gastroenterol 10: 2140-2144, 2004.

23. Zheng HH, Li YL, Yang HK, Lv YH and Gong CL: The empirical study of the mice-transplanted tumor of S-180 mediated by D-Limonene. Anatomy Research 1: 25-28, 2008. 